\title{
Questions de forme et de genre en traduction poétique $^{1}$
}

\author{
Ildikó Szilágyi \\ École Supérieure de Nyíregyháza \\ Hongrie
}

Résumé : Dans cet article nous nous intéressons à la poésie française moderne et contemporaine sous l'angle de sa traduction vers le hongrois. On commence par évoquer les traditions hongroise et française dans le domaine de la traduction poétique. On s'intéresse ensuite à la possibilité de traduction du verset, genre poétique moderne n'ayant même pas de nom en hongrois. On continue par s'interroger sur le retour au vers de certains poètes français contemporains et sur les difficultés que rencontrent les traducteurs hongrois voulant faire sentir la portée de ce retour. Pour finir, on évoque les contraintes oulipiennes à propos de la traduction du recueil Quelque chose noir de Jaques Roubaud.

Mots-clés: poésie française moderne et contemporaine, traduction poétique, « transfert » des schémas métriques, nouveaux genres poétiques

Abstract: The present study examines modern and contemporary French poetry from the point of view of its translation into Hungarian. Firstly, the French and Hungarian traditions of poetic translation are summarized briefly. Then the possibility of translation of "verset" (long verse), not recognized in Hungarian poetry as a modern poetic genre, is discussed. Particular attention is called to the return of several contemporary French poets to traditional versification, and to the difficulties of Hungarian translators to suggest the importance of this return. Finally, the Hungarian translation of Roubaud's volume of poetry (Quelque chose noir) invites reflection on the constraints of Oulipo.

Keywords: modern and contemporary French poetry, poetic translation, "transfer" of metrical schemes, new poetic genres

\section{Introduction}

Dans cet article nous nous intéressons à la poésie française moderne et contemporaine sous l'angle de sa traduction vers le hongrois. Notre travail s'inscrit, d'un côté, dans les recherches sur la

\footnotetext{
${ }^{1}$ La rédaction du présent article a été soutenue par la bourse Bolyai János Kutatási Ösztöndíj del'Académie Hongroise des Sciences (MTA).
} 
traduction poétique, aujourd'hui de plus en plus intensives, d'autre côté dans l'approche linguistique de la poésie, insistant sur la spécificité du langage poétique. Nous nous efforcerons de mettre en évidence la créativité de l'activité traductrice et d'évaluer les difficultés liées à la traduction de telle ou telle forme poétique. Nous considèrons les textes traduits comme oeuvres à part entière dont il convient d'examiner le mode particulier de signifier (Meschonnic 1999, 125) ${ }^{2}$.

Nous commencerons par évoquer brièvement les traditions hongroise et française dans le domaine de la traduction poétique, en particulier dans leur rapport avec la forme des poèmes traduits. Nous nous intéresserons ensuite à la possibilité de traduction du verset, apparu en France au début du XX $\mathrm{XX}^{\mathrm{e}}$ siècle. La difficulté de traduction vient en partie du fait que ce genre poétique n'a même pas de nom en hongrois. Les traducteurs hongrois du verset de Claudel et de SaintJohn Perse devraient donc créer dans la langue d'arrivée les conditions de son accueil. Nous continuerons par nous interroger sur le retour au vers (c'est-à-dire à la versification traditionnelle et aux formes fixes) des poètes français contemporains et sur les difficultés que rencontrent les traducteurs hongrois voulant faire sentir la portée de ce retour, considéré parfois par les critiques français comme «un retour en arrière », « un oubli-de-la-modernité » ou « une régression »3. Pour finir, nous évoquerons les controverses sur l'intraduisibilité de la littérature oulipienne et les contraintes à travers la traduction récente (2010) du recueil Quelque chose noir de Jaques Roubaud.

\section{La traduction poétique en Hongrie}

En Hongrie, la littérature traduite fait partie intégrante de la littérature nationale. La valorisation symbolique de l'apport de l'étranger à travers les traductions remonte à plusieurs siècles (Józan 2009, 13-63). La littérature et en particulier la poésie hongroises définissent leur identité par rapport à la littérature mondiale. La quantité extrêmement élevée des traductions s'explique par l'isolement linguistique du pays (le hongrois étant une langue finno-ougrienne agglutinante). Il s'agit non seulement de rendre accessible au lecteur hongrois la poésie étrangère, mais aussi d'élever la poésie hongroise à un niveau supérieur.

La traduction des poèmes étant considérée un art, le traducteur poétique jouit en Hongrie d'un prestige social considérable. La situation a commencé à changer au cours de ces dernières années, d'autant plus que la priorité est donnée (au détriment de la poésie) aux oeuvres en prose qui trouvent plus facilement des éditeurs et des traducteurs.

Depuis au moins le $\mathrm{XIX}^{\mathrm{e}}$ siècle, traduire de la poésie signifie en Hongrie traduire dans le respect de la forme. La langue hongroise

2 «L'objectif de la traduction n'est plus le sens, mais bien plus que le sens, et qui l'inclut : le mode de signifier. "

3 Critiques adressées respectivement à Louis Aragon et à Jacques Réda. 
permet d'écrire des vers mesurés selon trois systèmes de versification : syllabique, quantitatif et accentuel. L'opposition de longueur vocalique rend techniquement possible l'adaptation des mètres gréco-latins. C'est ainsi que les traducteurs peuvent fixer comme objectif de reproduire la forme originale, en fait de créer des mètres "équivalents " aux originaux. Le "transfert » des schémas métriques n'est pourtant pas sans problèmes. Comme l'écrit Christine Lombez (2003, 357), " certains mètres (hexamètre dactylique, alexandrin, distique élégiaque) sont étroitement liés à des traditions littéraires, de sorte que l'on ne saurait impunément traduire sans s'être au préalable interrogé sur le poids culturel qu'ils sont susceptibles de véhiculer ».

La naturalisation des différents types de vers, de strophe et de forme fixe, inexistants auparavant dans la prosodie hongroise, correspondait à la volonté affichée de moderniser la poésie hongroise et de l'intégrer dans la poésie mondiale.

Les prises de position concernant la fidélité formelle diffèrent considérablement d'une époque et d'une culture à l'autre. La pratique centenaire hongroise qui traduit la forme régulière par une forme régulière est loin d'être générale à l'étranger (par exemple en France). Dans sa postface aux poèmes choisis d'Attila József $(1998,154)$, György Timár explique ainsi la "méfiance » des Français envers l'observation des règles formelles sévères : « la nécessité absolue de faire revivre dans notre propre langue les poésies d'autrui n'existe pas dans les pays qui développent une certaine autarcie linguistique et littéraire. »

En France la reproduction de la forme originale était une pratique plutôt rare à côté de la traduction des poèmes réguliers en prose ou en vers libre. Les critiques hongrois aiment se référer - d'une manière bien exagérée - au rôle joué par l'Anthologie de la poésie hongroise (Seuil, 1962) dans l'introduction de la fidélité formelle en France. Le rédacteur de l'anthologie, László Gara, a réussi à faire accepter par les poètes-traducteurs de rendre les poèmes hongrois en respectant la forme des originaux. Cette anthologie peut être considérée comme un hommage des poètes français à la Hongrie, après la répression soviétique de l'insurrection du pays en 1956.

L'intérêt grandissant des traducteurs français pour les questions formelles s'explique plutôt par une plus grande ouverture de la France envers l'étranger, mais aussi par le nombre de plus en plus élevé de poètes français se mettant à la traduction poétique ${ }^{4}$. Tandis qu'en Hongrie il est tout à fait naturel que les meilleurs poètes, presque sans exception, accomplissent une oeuvre importante de traduction, en France « le cas des poètes traducteurs est sans doute le plus atypique » (Lombez 2003, 361). Eugène Guillevic $(1977,25)$ ne manque pas de s'indigner contre cette situation: "On ne voit pas pourquoi et

\footnotetext{
4 Les activités de traducteur et de créateur peuvent s'influencer mutuellement. Pour ne citer qu'un exemple célèbre : il ne fait pas de doute que les traductions baudelairiennes des œuvres d'Edgar Poe ont joué un rôle important dans la formation de l'esthétique du poète français.
} 
comment des diplômes universitaires ou d'autres titres conféraient à quelqu'un le don poétique, le don de manier le langage de telle façon que celui-ci accède à la dignité du poème ».

Les poètes-traducteurs de ces dernières décennies (Eugène Guillevic, Jean Rousselot, Yves Bonnefoy, Philippe Jaccottet, André du Bouchet, Denis Roche, parmi bien d'autres) semblent accepter qu' « on ne peut, on ne doit traduire les vers qu'en vers » (Etkind 1982, 276). Ceci dit, ils se soucient rarement d'observer rigoureusement toutes les contraintes formelles de l'original. En effet, «la question du vers ne tient pas tant, malgré l'apparence, aux contraintes du mètre et de la rime, qu'au principe de cohérence [...] d'une poétique du texte " (Meschonnic 1999, 162). Le traducteur doit reconnaître le travail du rythme prosodique mis en oeuvre dans le poème pour pouvoir en rendre l'organisation linguistique, rhétorique et poétique.

Pour revenir à la pratique de la traduction poétique hongroise, notons que, depuis les années 1980, plusieurs poètes-traducteurs (György Somlyó, József Tornai) font remarquer qu'à cause des problèmes d'équivalence des systèmes de versification «la fidélité formelle que l'on aime déclarer conventionellement absolue n'est valable, même dans les cas les moins problématiques [...], qu'avec des restrictions » (Somlyó 1981, 123). Malgré cela, les quelques tentatives récentes refusant le tabou de la fidélité formelle (traduisant en vers blancs des vers rimés, par exemple) se heurtent encore en Hongrie à l'incompréhension de la plupart des critiques et des lecteurs. (Quant à nous, nous ne sommes pas pour le respect servile de tous les éléments formels de l'original, mais nous ne pouvons pas accepter, même théoriquement, comme le fait Paola Masseau (2012, 228), la traduction en prose du « Cimetière marin » de Valéry.)

Un autre problème soulevé par Somlyó et qui concerne notre sujet de plus près est le fait que

dans la poésie moderne la forme du poème doit être cherchée, trouvée et recréée en dehors des éléments traditionnels de la poésie. Ici, on commence à se trouver dans le vide avec notre tradition reprise et pratiquée à un niveau élevé. $(1981,139)$

Les nouveaux genres poétiques (poème en prose, vers libre, verset) ont en commun d'être redevables aux traductions poétiques. Les premiers poèmes en prose se présentent sous forme de pseudo-traductions. Les traductions françaises de Shakespeare servent de modèle à la versification assouplie du drame romantique. Les premières traductions de Walt Whitman sont parmi les influences éventuelles qui ont abouti au vers libre. Le verset moderne s'inspire (à travers les traductions) de son modèle biblique.

Diffusés et acceptés d'abord comme formes, ils ne seront admis comme genres autonomes qu'au bout d'une période de légitimation plus ou moins longue. Ils se constituent en marge des genres traditionnels, rendant problématique la distinction même de la prose et 
du vers (identifié traditionnellement à la poésie). Le brouillage métrique s'accompagne donc d'un brouillage des genres. Il faut néanmoins éviter le danger de se contenter d'une traduction littérale. Certes, les traits formels du vers libre et du verset sont plus cachés, moins évidents que les contraintes d'un sonnet par exemple. Le traducteur doit devenir plus attentif au travail qu'accomplit le poète sur la forme de son poème, pour mobiliser ensuite toutes les ressources de la langue d'arrivée.

\section{La traduction du verset (Claudel, Saint-John Perse)}

Dans ce qui suit, nous nous proposons d'examiner les difficultés de traduction du verset, de loin le genre poétique moderne le moins étudié. Le travail du traducteur est d'autant plus difficile que l'autonomie $d u$ verset n'est pas unanimement reconnue par les critiques. D'aucuns l'admettent comme l'une des écritures possibles du poème en prose, d'autres (les plus nombreux) le rangent parmi les variantes du vers libre. Situé à l'intersection de ces deux genres, le verset intègre certains de leurs traits caractéristiques.

À l'origine, on parle de verset pour nommer les divisions numérotées de la Bible et d'autres textes sacrés. Ce n'est que depuis le début du $\mathrm{XX}^{\mathrm{e}}$ siècle que le mot commence à désigner dans un sens profane un nouveau genre poétique. Paul Claudel l'utilise d'abord dans ses premières pièces de théâtre, ensuite dans les Cinq grandes odes (1910). L'influence de Claudel est palpable dans les recueils de jeunesse de Saint-John Perse dont Les Éloges (1911) qui paraissent encore sous son vrai nom : Alexis Saint-Léger Léger. Nos analyses se basent sur ces deux recueils et sur leur traduction hongroise.

Le terme français « verset » est traduit en hongrois en " poème biblique » (se référant à son origine), ou bien en "vers libre de grande longueur », mais il arrive aussi que le mot français soit gardé dans le texte hongrois (mis en italique).

Les Cinq grandes odes de Paul Claudel sont traduites en hongrois en 2005 à l'occasion du $50^{\mathrm{e}}$ anniversaire de la mort du poète. (En vérité, le traducteur, Ferenc Szabó ne traduit intégralement que la deuxième ode, ne donnant que des extraits des quatre autres odes.) Ferenc Szabó (1931-) est membre de l'ordre religieux de la Compagnie de Jésus, poète, essayiste, traducteur, théologien. Par ses études littéraires, philosophiques et théologiques, ainsi que par ses traductions de Claudel et de Pierre Emmanuel, il contribue à faire connaître en Hongrie l'esthétique catholique française. (Notons que le Choix d'œuvres de Paul Claudel, reprenant essentiellement la traduction des pièces de théâtre, n'apparaît qu'en 1982. L’idéologie politique du régime communiste ne favorisait pas la diffusion de l'œuvre claudélienne en Hongrie.)

Lorsqu'il obtient en 1960 le prix Nobel de littérature, Saint-John Perse, alors âgé de 73 ans, est presque inconnu en Hongrie. Ses poèmes 
de jeunesse ne sont publiés en hongrois qu'en 2000 sous le titre Dicséretek [Éloges] dans la traduction de Róbert Bognár. La traduction hongroise contient en fait trois recueils poétiques : «Éloges » évoque la nostalgie de l'enfance, La Gloire des Rois parle de la culture plus ou moins réaliste d'une tribu, tandis que Anabase fait apparaître une mythologie tout à fait fictive. Il est à noter que ce dernier recueil (Anabase) a été traduit en plusieurs langues quelques années seulement après sa publication (1924) : la traduction allemande (par Groethuysen et Walter Benjamin) est introduite par Hofmannstahl (1929), c'est T. S. Eliot qui le traduit en anglais (1930), et Ungaretti - en italien (1931). Rappelons que la traduction hongroise ne date que de 2000. Auparavant un seul recueil poétique de Saint-John Perse (1969) apparaît en hongrois : Bóják [Amers], "un poème ou une épopée " selon le traducteur István Vas.

Róbert Bognár (1947-) est connu surtout comme traducteur littéraire français-hongrois. Il a traduit une cinquantaine de livres et presque autant de pièces de théâtre, des oeuvres de style très différent : les Chants de Maldoror de Lautréamont, les drames de Molière, d'Ionesco et de Genet, les Exercices de style de Queneau, les romans de Boris Vian et de Balzac.

Les recueils de Claudel et de Saint-John Perse présentent, d'un point de vue formel, à l'époque de leur publication (respectivement en 1910 et 1911), "une nouveauté extraordinaire différant de la versification traditionnellement ancienne et de la versification traditionnellement moderne » (Saint-John Perse 1969, 16). On vient de citer l'opinion d'István Vas qui dans la préface écrite à sa traduction des Amers s'attarde longuement à expliquer la nouvelle forme poétique utilisée par Saint-John Perse sans mentionner une seule fois le terme de «verset». La traduction hongroise des Cinq grandes odes de Claudel est également dotée d'une introduction, mais cette dernière ne traite de questions formelles qu'en passant.

Le décalage temporel très important (presque cent ans) entre la publication des traductions hongroises et celle des recueils originaux ne permet pas de faire sentir l'effet produit sur les premiers lecteurs. La versification "libre et déferlante » (16) de Claudel et de Saint-John Perse a perdu sa «nouveauté extraordinaire». Le lecteur hongrois contemporain peut se référer outre les nombreux exemples étrangers, aux poètes comme Milán Füst ou Ferenc Juhász. (Malgré les similitudes formelles et thématiques, le terme " verset » n'est pourtant pas évoqué à propos de leur poésie par les critiques hongrois.)

Le traducteur des Cinq grandes odes, Ferenc Szabó, désirait satisfaire avant tout la contrainte de la fidélité théologique. Ses traductions sont proposées en tant que thèmes d'exercice spirituel. Cette motivation pédagogique peut expliquer certains choix du traducteur, mais soulève la question de l'équivalence fonctionnelle. Le texte-cible ne joue plus exactement le même rôle auprès du public-cible que le texte-source jouait auprès du public-source. 
C'est ainsi que le maintien des caractéristiques formelles ne représente pas pour Szabó une priorité. Dans certains cas, il change l'organisation strophique des poèmes $(2005,101,106$, etc.). Ce qui est plus important, c'est que la disposition des lignes diffère souvent de la mise en forme originale. Dans l'exemple suivant il contracte en une seule unité typographique (un seul verset) trois lignes originales. Le contenu reste intact, mais le rythme ralentit.

Quelle

Porte m'arrêterait? quelle muraille ? L'eau

Odore l'eau, et moi je suis plus qu'elle-même liquide! (Claudel 1957, 237)

Milyen kapu állíthatna meg? milyen fal? A víz vízszagot áraszt, és én magam folyékonyabb vagyok nála! (Claudel 2005, 100)

La fin d'un verset biblique correspond toujours à une fin de groupe syntaxique et sémantique, le plus souvent coïncidant avec la fin d'une phrase. Le verset claudélien diffère sur ce point de son modèle biblique. Peut-être le traducteur voulait-il éviter l'enjambement, inconnu dans les textes bibliques, rendant ainsi plus équilibré le poème claudélien perturbé, tourmenté. Il est à noter que le traducteur ne contracte plusieurs lignes en une seule que dans les cas où cette réduction fait disparaître l'enjambement.

Contrairement au vers libre, le verset - en tant qu'unité typographique - ne se définit pas par le retour à la ligne. Il se poursuit le plus souvent bien au-delà de la marge droite. La possibilité de l'enjambement due à la présence du blanc final (que le poète en fasse un usage fréquent ou non) est donc un indice important qui permet d'apparenter le verset au vers et de le différencier du paragraphe. Dans l'exemple cité, le pronom interrogatif « quelle », isolé sur une ligne, ou le sujet, dissocié de son prédicat («l'eau / odore l'eau») se trouvent en position accentuée, l'enjambement y remplit une fonction suspensive et emphatique. On ne peut pas le supprimer sans perte considérable.

La traduction de Róbert Bognár suit fidèlement la disposition typographique du texte persien, il fait même attention à rendre les fréquents enjambements de Saint-John Perse à leur place originale.

La question de l'enjambement nous amène à la problématique des caractéristiques «métriques ». Il est habituel d'identifier des segments syllabiques rappelant des types de vers familiers dans les versets dits "métriques » de Saint-John Perse. Quant au verset claudélien, qualifié souvent de verset «cadencé », il repose sur la succession des groupes accentuels distribués selon un ordre croissant ou décroissant.

Le repérage et la reproduction de ces éléments d'une manière plus systématique et consciente aurait enrichi les traductions hongroises. Les groupes binaires (principalement de six, de huit et de douze syllabes), dont la dominance est tout à fait remarquable chez Saint-John 
Perse, ne se retrouvent qu'accidentellement dans la traduction hongroise des paragraphes plus longs. Il est intéressant de remarquer que les deux traducteurs ont très fréquemment recours à l'allitération (plus fréquemment même que Claudel ou Saint-John Perse), voulant peut-être compenser par ce moyen les éléments de versification traditionnelle qui réapparaissent de temps en temps dans les originaux. "Un peu de ciel bleuit au versant de nos ongles » (Saint-John Perse 1960, 36) est traduit en « Kevéske ég kéklik körmünk fonákján » (Saint-John Perse 2000, 42) où il est aisé d'apercevoir l'allitération en [k].

Les répétitions et constructions parallèles (caractéristiques principales du verset hébraïque suivant les recherches de Robert Lowth) se manifestent à tous les niveaux linguistiques. Outre les récurrences phoniques, les deux recueils présentent de nombreux cas de répétitions lexicales, morphologiques, syntaxiques et sémantiques. La comparaison des recueils originaux et des traductions montre que les traducteurs accordent aux structures répétitives et parallèles une importance particulière. Ils y voyaient les traits distinctifs des poèmes à traduire qu'ils s'efforçaient de rendre dans leurs traductions.

Le choix du verset chez Claudel est étroitement lié à la quête spirituelle qui s'exprime dans ses textes nourris d'allusions liturgiques. La parenté avec l'éloquence sacrée ne sera pas toujours si évidente chez les poètes contemporains, introduisant dans leurs poèmes le quotidien, le banal, la dérision et l'autoréflexion.

Les poètes français contemporains écrivant en versets (Benoît Conort, James Sacré etc.) sont pratiquement inconnus en Hongrie. La traduction d'un verset de Guy Goffette est publié dans l'anthologie intitulée $A$ hatlapú rigó [Le merle à six côtés], présentant des poètes français-belges contemporains $(2004,23-26)$. La référence intertextuelle du titre, «Le prunier de Claudel », montre que le choix du verset claudélien comme genre poétique n'est qu'un jeu ironique. Il s'agit d'une "dilecture ", procédé de distanciation cher à Goffette. Le traducteur doit donc faire sentir l'irrévérence du poète contemporain envers l'héritage accablant du grand prédécesseur.

\section{La traduction des poemes du « nouveau lyrisme » (Goffette)}

Jusqu'aux années 70-80, il n'est pas exagéré de parler dans la traduction poétique hongroise d'une dominance de la poésie française et, plus particulièrement, de la poésie française moderne. Dans les trois dernières décennies, l'attention des traducteurs tourne plutôt vers les langues anglaise et (en moindre mesure) allemande. Il est significatif que l'anthologie de György Somlyó (Hallomás [Ouï-dire]), présentant trente-six poètes français contemporains, datée de 1971 ne sera suivie par une nouvelle anthologie de poésie contemporaine qu'en 1995 ( $A$ látogatás [La visite]). Krisztina Tóth, la rédactrice de cette anthologie, choisit plus de cent cinquante poèmes, publiés en recueil dans les 
années 80 et au début des années 90 en France5. Les quinze traducteurs, dont la rédactrice elle-même, sont presque sans exception des poètes, en même temps, ils représentent la nouvelle génération de traducteurs littéraires dans le domaine français-hongrois.

D'un point de vue formel, les poèmes de l'anthologie se divisent en trois grands groupes : des vers libres (la grande majorité), poèmes en prose ou proses lyriques et des poèmes reprenant des éléments de versification traditionnelle.

Les poètes du nouveau lyrisme des années 1980 retournent à l'alexandrin, ou au moins à ses "décombres" (Lackfi 2008, 106). Ils sont devancés par Jacques Réda, Philippe Jaccottet, Yves Bonnefoy de la génération précédente. Il s'agit d'un retour conscient, d'un choix esthétique délibéré de la part des poètes. János Lackfi attire l'attention sur un problème de traduction important à propos de ce nouveau phénomène de la poésie française contemporaine :

il se révéla que ce que la grande partie de la poésie française considérait comme une contrainte insupportable, n'était autre que la nouvelle forme de la liberté. Celui qui ne connaît ou au moins ne sent pas cela, n'est pas capable de traduire d'une manière adéquate même les textes les plus simples. (107)

Il est important d'insister sur ce point, parce que le lecteur hongrois ne voit pas forcément dans ce retour " une percée révolutionnaire " (106), étant donné que presque les deux tiers des poèmes hongrois parus en anthologies ou en recueils depuis 1945 sont de versification régulière.

Il faut reconnaître que les poètes français du nouveau lyrisme ne retournent pas au vers métrique du XIX ${ }^{\mathrm{e}}$ siècle. Les poèmes de systèmes métrique, strophique, rimique également réguliers restent plutôt rares. Il est plus fréquent qu'un vers libre utilise librement les éléments de versification traditionnelle.

Le regain d'intérêt pour le vers dans la poésie contemporaine s'accompagne chez plusieurs poètes par la redécouverte des poèmes à forme fixe, avant tout par celle du sonnet.

Dans ce qui suit, nous illustrerons les problèmes de traduction liés à la reprise des éléments de versification traditionnelle par les poèmes de Guy Goffette (1947-), traduits en hongrois.

Les 16 poèmes du poète français-belge publiés dans l'anthologie de 1995 seront repris et complétés par d'autres dans le recueil de traduction intitulé Vízhalász [Le Pêcheur d'eau] en 1997.

\footnotetext{
5 Guy Gofette, Didier Pobel, Gérard Macé, Dominique Sampiero, Christian Bobin, JeanPierre Chambon, Emmanuel Moses, Alain Naud, Paul Le Jéloux, Jean-Charles Vegliante, Hédi Kaddour, Paul de Roux, Philippe Delaveau, Jean-Pierre Lemaire, Christian Doumet, Bruno Grégorie, Jean-Baptiste de Seynes, François Boddaert, Petr Král, Serge Pey, Liliane Giraudon, Jean-Luc Parant, Yvon Le Men, Jean Monod, André Velter, Jean-Michel Maulpoix, Yves Charnet, Martine Broda, Jacques Darras, Lionel Ray, Ludovic Janvier.
} 
Quelles sont les caractéristiques formelles les plus importantes de ces poèmes et de leur traduction? On peut tenter de répondre à cette question dans un premier temps par le repérage des éléments traditionnels (au niveau de l'organisation strophique, métrique et rimique). Ayant perdu tout caractère obligatoire, l'identification de ces éléments n'est plus possible dans un contexte libre que par référence culturelle au modèle classique.

Les traducteurs (Krisztina Tóth, András Imreh et János Lackfi) suivent de près la disposition typographique évoquant les formes traditionnelles (dont le sonnet). Ils se soucient de rendre non seulement les allitérations et assonances compensant l'absence des rimes finales, mais aussi les nombreux enjambements et les constructions syntaxiques relâchées.

Pour un lecteur moderne, déjà habitué à une variété extrême dans la longueur des vers libres, les poèmes de Goffette donnent l'impression d'une certaine régularité. On peut y reconnaître, par delà la liberté métrique, la référence, bien qu'un peu floue, à l'alexandrin. En effet, beaucoup de vers tournent autour de 12 syllabes, ils les atteignent parfois, ou comptent une syllabe de plus, ou une syllabe de moins. Tout en gardant une apparence extérieure rassurante, les poèmes dissimulent une grande diversité dans leur structure de détail. La difficulté de la traduction vient du fait qu'il faut donner une idée de cette diversité, tout en gardant la référence à la régularité. On peut dire d'une façon générale que les traducteurs essaient de rendre les éléments traditionnels. Il arrive même que la traduction hongroise devienne plus régulière que l'original. Le quatrain suivant («Un peu d'or dans la boue V ») se compose à l'origine de 12, 10, 11 et 5 syllabes.

On dit : le soleil après la pluie, la mer

après la montagne, l'amour après

et partir, partir. Demain, quand tout sera,

quand tout aura, quand. (Goffette 1991, 185)

Dans la traduction hongroise de Krisztina Tóth les vers s'allongent pour compter 12, 12, 12 et 8 syllabes. Il s'agit de la version de l'anthologie $(1995,8)$. Il est intéressant de noter que dans le recueil de traduction Vízhalász (1997, 10), ce même poème est retraduit: avec la suppression du mot « holnap » [demain], le troisième vers ne comptera plus que 10 syllabes.

\section{La traduction des poèmes à contraintes (Roubaud)}

Pour terminer, nous nous intéresserons à la traduction de la poésie de l'Oulipo (Ouvroirs de Littérature Potentielle) à travers la traduction du recueil Quelque chose noir de Jacques Roubaud (1986). Roubaud est l'un des poètes français contemporains les plus importants, titulaire de doctorat d'État en mathématiques et en littérature française, membre de l'Oulipo (comme Raymond Queneau, 
Georges Perec, Italo Calvino et d'autres). Les oulipiens sont souvent évoqués dans les débats sur la traductibilité de la poésie. Considérés " comme créateurs de textes intraduisibles ou pour le moins très difficiles à traduire » (Seláf 2011, 26), ils ont suscité très peu de traductions en hongrois. La traduction de la poésie régulière, liée également par maintes contraintes, est pourtant très appréciée en Hongrie. Les deux sont des créations formalistes, reposant sur des contraintes strictes, fixées au départ.

Quelque chose noir, le recueil poétique le plus célèbre de Roubaud, commémore la mort prématurée de sa femme, la photographe Alix Cléo Blanchette. Levente Seláf en fait paraître la traduction intégrale en 2010sous le titre Vala mi : fekete. L'étrangeté du titre, due à la faute grammaticale («quelque chose noir» au lieu de «quelque chose de noir » ou «quelque chose noire »), est rendue par la fragmentation du premier mot, et par les deux points mis entre les deux mots.

Le recueil se compose de 82 poèmes ( 9 sections de 9 poèmes de 9 vers ou paragraphes, complétées par un poème final). Cette contrainte simple du chiffre 9 est aisément traduisible. Mais, pour le traducteur, "le doute persiste: a-t-il identifié toutes les contraintes cachées ? N'est-il pas passé à côté d'un principe d'organisation qu'il eût été nécessaire de reproduire dans la traduction ? 》 (Seláf 2011, 33).

Les textes du recueil sont qualifiés de poèmes par Roubaud $(1986,85)$ : « ces phrases de neuf que je nomme poèmes ». Il n'est pas possible de rendre en hongrois la polysémie du mot « neuf » : il signifie le chiffre 9, mais aussi « nouveau », de plus, il rime avec « veuf ».

"Quelque chose va sortir du silence, de la ponctuation, du blanc remonter jusqu'à moi ", écrit Roubaud dans Dialogue (1986, 124). L'emploi très particulier des signes de ponctuation (l'absence fréquente de points finals ou la surabondance des virgules, par exemple), ainsi que la mise en page aérée par des blancs sont des marques distinctives de la poésie de Roubaud. Loin d'être un simple vide, le blanc a, chez le poète, une réalité physique s'opposant au noir. Le traducteur doit donc accorder une attention particulière à ces éléments typographiques.

Dans ce recueil, situé à l'intersection de la poésie et de la prose, le poème " Énigme ", écrit en alexandrins bien césurés (à quelques exceptions près) et partiellement rimés, se détache avec éclat. On en cite la première séquence, suivie par sa traduction hongroise :

Sans être maintenant sans lieu sans poids......sans dire (6-6)

Sans parenté vivante accrochée au regard (6-6) (Roubaud 1986, 97)

Léttelen súlytalan helytelen kimondatlan (6-7)

A szemre tapadó élő rokon nélkül (6-6) (Roubaud 2010, 83)

L'espacement interne (le blanc typographique sépare des unités sur une même ligne) interrompt les phrases et modifie le rythme de la lecture.

Traditionnellement, l'équivalent hongrois de l'alexandrin français est un vers iambique de 12 syllabes (en cas de rime masculine), 
ou un vers trochaique de 13 syllabes (en cas de rime féminine), césuré après le premier hémistiche. Le traducteur, voulant mettre en relief le caractère exceptionnel de ce poème (presque) régulier, renoue avec la tradition de la traduction hongroise, ayant soin à reproduire même cette syllabe surnuméraire (avec quelques exceptions).

\section{Conclusion}

Malgré la «crise de vers » et l'éclatement des genres au $\mathrm{XX}^{\mathrm{e}}$ siècle, les questions de forme et de genre restent d'actualité. Tout traducteur de poésie moderne et contemporaine doit prendre en considération le fait que les genres nouveaux ont également quelques traits pertinents qu'il faut respecter, et dont il convient de trouver des équivalents dans la langue " cible ». Faute de repères traditionnels, l'identification et la reproduction de ces caractéristiques sont loin d'être évidentes. Le traducteur aura alors " un triple rôle: critique, traducteur et créateur " (Oseki-Dépré 2012, 13), tandis que la pratique de la traduction poétique se révélera un important outil de critique littéraire.

\section{Références bibliographiques}

Etkind, Efim. Un art en crise : essai de poétique de la traduction poétique. Lausanne : L'Age D'Homme, 1982.

Guillevic, Eugène. Mes poètes hongrois. Budapest : Éditions Corvina, 1977 [1967].

Józan, Ildikó. Baudelaire traduit par les hongrois vers une théorie de la traduction. Paris : Presses Sorbonne Nouvelle, 2009.

József, Attila. Complainte tardive. Poèmes choisis. Choix, adaptations et postface par Georges Timár. Budapest : Balassi Kiadó, 1998.

Lackfi, János. «A fordítás betegség, akár a szerelem » [La traduction est une maladie comme l'amour]. In: Jeney, Éva - Józan, Ildikó (red.) 2008. Nyelvi álarcok. Tizenhárman a fordításról [Masques langagiers. Treize sur la traduction]. Budapest : Balassi Kiadó, 2008 : 80-112.

Lombez, Christine. "Traduire en poète. Philippe Jaccottet, Armand Robin, Samuel Beckett ». Poétique, Seuil, no 135, 2003 : 355-379.

Masseau, Paola. Une traductologie de la poésie est-elle possible? La traduction du poème "toujours recommencée ». Paris : Éditions Publibook, 2012.

Meschonnic, Henri. Poétique du traduire. Paris : Verdier, 1999.

Oseki-Dépré, Inès. « Remarques sur la traduction de la poésie ». Belas Infiéis, v. 1, no 2, 2012: 7-18.

Seláf, Levente. "Traduction des textes à contrainte: méthodes et expériences hongroises ». Hungarian Studies, Akadémiai Kiadó, vol. 25/ 1, 2011 : 25-36.

Somlyó, György. "Két szó között. Megjegyzések a fordítás poétikájához » [Entre deux mots. Remarques sur la poétique de traduction]. In : Bart, István - Rákos, Sándor (red.). A müfordítás ma [La traduction littéraire aujourd’hui]. Budapest : Gondolat, 1981 : 102-146. 


\section{Corpus}

Claudel, Paul. Kantáta három hangra és más költemények [Cantate à trois voix et autres poèmes]. Traduit par Ferenc Szabó. Debrecen : Új Ember Kiadó, 2005.

Claudel, Paul. Oeuvre poétique. Bibliothèque de la Pléiade. Paris : Gallimard, 1957 : 221-292.

Goffette, Guy. La vie promise. Paris : Gallimard, 1991.

Goffette, Guy. Vízhalász [Le Pêcheur d'eau]. Poèmes choisis et traduits par Krisztina Tóth, András Imreh, János Lackfi. Budapest : Széphalom Könyvmühely, 1997.

Lackfi, János (red.). A hatlapú rigó. Kortárs francia-belga költők [Le merle à six côtés. Poètes français-belges contemporains]. Budapest : Nagyvilág, 2004.

Roubaud, Jacques. Quelque chose noir. Paris : Gallimard, 1986.

Roubaud, Jacques. Vala mi: fekete. Traduit par Levente Seláf. PozsonyBudapest : Kalligram, 2010.

Saint-John Perse. Bóják [Amers] Traduit et préface de István Vas. Budapest : Európa, 1969.

Saint-John Perse. Dicséretek. Királyok dicsősége. Anabázis [Éloges. La Gloire des Rois. Anabase]. Noran Kiadó, 2000.

Saint-John Perse. Éloges suivi de La Gloire des Rois, Anabase, Exil. Paris : Gallimard, 1960.

Somlyó, György (red. et trad.) Hallomás [Ouï-dire]. Budapest : Európa, 1971. Tóth, Krisztina (red.). A látogatás. Kortárs francia költők [La visite. Poètes français contemporains]. Budapest - Pécs : Jelenkor Kiadó, 1995. 